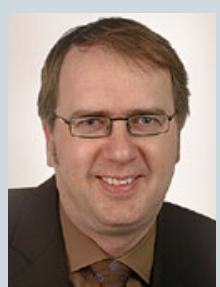

Prof. Dr. med. Dr. phil.

Stefan Evers

Krankenhaus

Lindenbrunn,

Coppenbrügge

\title{
Neue Studien zum Kopfschmerz
}

\author{
Die Studienübersicht zum Thema Kopfschmerz geht schwerpunktmäßig auf die Migräne ein, aber auch \\ Clusterkopfschmerz und Trigeminusneuralgie dürfen nicht fehlen. Neben Medikamenten werden überdies \\ Maßnahmen wie Stimulationsverfahren oder Lichttherapie kritisch bewertet.
}

\section{Gibt es eine Evidenz für die Stimulation des N. vagus bei primären Kopfschmerzen?}

\section{Die transkutane Vagusstimulation am Hals zur Prophylaxe der chronischen Migräne und des chronischen Clusterkopfschmerzes zeigt einen positiven Trend, muss aber weiter in Studien untersucht werden.}

Z ur Prophylaxe der chronischen Migräne und des chronischen Clusterkopfschmerzes werden neben Medikamenten auch Stimulationsverfahren untersucht, darunter die transkutane Vagusstimulation am Hals. Offene Fallberichte und Studien aus letzter Zeit weisen auf eine Wirksamkeit hin, in einer Studie mit Vagusstimulation mittels Ohrelektrode wurde sogar ein signifikanter Effekt bei chronischer Migräne beobachtet, in einer anderen Studie auch beim chronischen Clusterkopfschmerz. Jetzt wurden in den USA zwei prospektive doppelblinde Studien (chronische Migräne und Clusterkopfschmerz) durchgeführt. Die Patienten wurden nach einer Beobachtungsphase von einem Monat in zwei Gruppen eingeteilt: In der Verumgruppe stimulierten die Patienten zweimal täglich zwei Minuten oder bei Bedarf den N. vagus rechts am
Hals. Die Kontrollgruppe stimulierte identisch, aber ohne Strom. Der doppelblinden folgte eine offene Phase.

Bei chronischer Migräne [1] konnten 48 Patienten ausgewertet werden. Nach zwei Monaten nahm die Zahl der Kopfschmerztage in der Stimulationsgruppe um 1,4 Tage und in der Kontrollgruppe um 0,2 Tage ab. In der offenen Weiterbehandlung kam es nach vier Monaten zu einer Abnahme der Kopfschmerztage um 5,8, nach sechs Monaten um 6,7 und nach acht Monaten um 7,9 pro Monat. Relevante Nebenwirkungen traten nicht auf. Der Effekt war nicht signifikant.

Beim Clusterkopfschmerz [2] wurden 150 Patienten mit akuter Attacke behandelt. Eine Response hatten 26,7\% nach Vagusstimulation, $15,1 \%$ nach Scheinstimulation. Dieser Unterschied war nicht signifikant. Allerdings zeigte sich in der Untergruppe des episodischen Cluster- kopfschmerzes im Gegensatz zum chronischen Clusterkopfschmerz eine signifikant erhöhte Responderrate. Auch beim Clusterkopfschmerz verursachte die Vagusstimulation keine relevanten Nebenwirkungen.

\section{Kommentar}

Diese beiden Studien zeigten keinen signifikanten Effekt der Vagusstimulation in der doppelblinden Behandlungsphase bei beiden Indikationen (obwohl eine Verblindung hier schwer ist, die Stimulation erzeugt nämlich bei vielen ein Vibrieren am Hals). Ein gewisser Trend kann jedoch nicht ausgeschlossen werden. Daher muss die transkutane nicht invasive Vagusstimulation bei chronischer Migräne (und beim Clusterkopfschmerz) in einer größeren und in ihrer doppelblinden Phase länger dauernden Studie wiederholt werden.

Prof. Dr. Dr. Stefan Evers

1. Silberstein SD et al. EVENT Study Group. Chronic migraine headache prevention with noninvasive vagus nerve stimulation: The EVENT study. Neurology 2016; 87: 1 - 10

2. Silberstein SD et al. ACT1 Study Group. Noninvasive vagus nerve stimulation for the acute treatment of cluster headache: findings from the randomized, double-blind, shamcontrolled ACT1 study. Headache 2016; 56 : 1317-32 